\title{
ANALYTICAL SOLUTIONS FOR THE INCOMPRESSIBLE VISCOUS FLOW WITHIN A RECTANGULAR DOMAIN
}

\author{
Masayuki OKABE \\ Mitsui Mining \& Smelting Company Ltd., Nihonbashi-Muromachi, Chuo-Ku, Tokyo 103, Japan \\ and \\ Noboru KIKUCHI \\ Department of Mechanical Engineering and Applied Mechanics, The University of Michigan, \\ Ann Arbor, MI 48109, U.S.A. \\ Received 28 January 1983
}

\begin{abstract}
Analytical solutions of the velocity, pressure and stream function are developed for the slow incompressible viscous fluid in a simple rectangular domain. All solutions can be applied to the boundary integral techniques as typical fundamental solutions.
\end{abstract}

\section{Introduction}

The finite element method has been zealously applied to the slow incompressible viscous flow (simply called the Stokes flow). One of the major difficulties arises through divergent-free constraints due to incompressibility. We have several alternatives; the mixed formulations [1-3], stream function methods [2,3] and reduced-integration penalty approximations [4-9]. In the second approach by the use of stream functions, we need some genuity to compute the pressure. In other finite element approximations, some smoothing procedures are necessary for the pressure $[8,9]$, although the higher order simplex interpolations [10] are always free from locking [11].

In the boundary element approach, on the other hand, the divergent-free conditions are realized quite easily [12]. However, another difficulty arises through incompressibility with regards to traction forces as shown in Appendix B. The usual boundary element approximations developed in linear elasticity [13] cannot be applied to the Stokes flow.

This paper is devoted to the analytical solutions for the steady Stokes flow within a simple rectangular domain, by which numerical techniques currently used can be examined directly. Not only the velocity and pressure but also the stream function are dealt with. Examples are then presented to which boundary element solutions in a least-square scheme are compared.

\section{Constitutive law}

We consider a slow incompressible viscous fluid in the domain $\Omega$ with isotropic homogeneous viscosity $\mu$. Let $\left(f_{x}, f_{y}\right)$ be a body force placed at a source point $\boldsymbol{x}_{s}=\left(x_{s}, y_{s}\right)$. Then the 
velocity $\boldsymbol{u}=(u, v)$ at the field point $\boldsymbol{x}=(x, y)$ satisfies the equilibrium equation of the form

$$
\begin{aligned}
& \mu \nabla^{2} u(x)+(\mu+1 / \varepsilon) \partial[\operatorname{div} u(x)] / \partial x+f_{x} \delta\left(x-x_{s}\right)=0, \\
& \mu \nabla^{2} v(x)+(\mu+1 / \varepsilon) \partial[\operatorname{div} u(x)] / \partial y+f_{y} \delta\left(x-x_{s}\right)=0 .
\end{aligned}
$$

Here $\nabla^{2}$ denotes the Laplacian, div designates the divergence, $\delta$ is the Dirac distribution and $\varepsilon$ is a parameter such that $\varepsilon \rightarrow 0^{+}$.

Let $p$ be the pressure defined by

$$
p(\boldsymbol{x})=-[\operatorname{div} \boldsymbol{u}(\boldsymbol{x})] / \varepsilon .
$$

Then (1) can be written as

$$
\begin{aligned}
& \mu \nabla^{2} u(x)-(1+\mu \varepsilon) \partial p(x) / \partial x+f_{x} \delta\left(x-x_{s}\right)=0, \\
& \mu \nabla^{2} v(x)-(1+\mu \varepsilon) \partial p(x) / \partial y+f_{y} \delta\left(x-x_{s}\right)=0 .
\end{aligned}
$$

In definition, the incompressible fluid is divergent-free such that

$$
\operatorname{div} \boldsymbol{u}(\boldsymbol{x})=0,
$$

while the pressure should not be zero, i.e. div $u(x)$ is $O(\varepsilon)$. At the limit of $\varepsilon \rightarrow 0^{+}$, (3) can simply be written as

$$
\begin{aligned}
& \mu \nabla^{2} u(x)-\partial p(x) / \partial x+f_{x} \delta\left(x-x_{s}\right)=0, \\
& \mu \nabla^{2} v(x)-\partial p(x) / \partial y+f_{y} \delta\left(x-x_{s}\right)=0,
\end{aligned}
$$

The boundary conditions shall be described for each problem.

We further define the stream function $\psi$ by

$$
\partial \psi / \partial y=u, \quad \partial \psi / \partial x=-v .
$$

REMARK 2.1. In the so-called penalty method, $\varepsilon$ is regarded as a penalty parameter which is positive and very small. However, in this paper, the solutions are given at the limit of $\varepsilon \rightarrow 0^{+}$ (i.e. independent of $\varepsilon$ ).

REMARK 2.2. For $\varepsilon>0$, (1) and (3) can be identified with the equilibrium equation in linear elasticity (plane strain), but not (5).

REMARK 2.3. If $\Omega$ is the infinite domain, then velocities at infinity should be zero. The natural boundary condition at infinity requires multiple body forces satisfying the force balance conditions of the form

$$
\sum_{i} f_{x}^{i}=0
$$


and/or

$$
\sum_{i} f_{y}^{i}=0
$$

Here superscript $i$ designates the body force number.

In this paper, for simplicity, we deal only with a point force $\left(f_{x}, f_{y}\right)$. If the force balance condition is needed in some direction, therefore, our solutions are inadequate. Notice that the linear superposition makes them adequate.

\section{Whole-space problem}

We first consider the whole-space $\Omega$. Here only the natural boundary condition at infinity is applied.

THEOREM 3.1. For the whole-space problem, the velocity and pressure at $\boldsymbol{x}$ can be expressed explicitly as

and

$$
\begin{aligned}
& u=A\left[-f_{x} \ln R-r_{y}^{0}\left(f_{x} r_{y}^{0}-f_{y} r_{x}^{0}\right) / R^{2}\right], \\
& v=A\left[-f_{y} \ln R-r_{x}^{0}\left(f_{y} r_{x}^{0}-f_{x} r_{y}^{0}\right) / R^{2}\right],
\end{aligned}
$$

Here

$$
p=C\left(f_{x} r_{x}^{0}+f_{y} r_{y}^{0}\right) / R^{2} .
$$

$$
\begin{array}{ll}
r_{x}^{0}=x-x_{s}, & r_{y}^{0}=y-y_{s}, \quad R=\left[\left(r_{x}^{0}\right)^{2}+\left(r_{y}^{0}\right)^{2}\right]^{1 / 2}, \\
A=1 / 4 \pi \mu, & C=1 / 2 \pi .
\end{array}
$$

$P R O O F$. In the case of $x \neq x_{s}$, it is easy to verify that the velocity and pressure by (8) and (9) satisfy the governing equations (5).

Let us introduce the polar coordinates $(R . \theta)$ of Fig. 1 origined at $x_{s}$ such that

$$
\cos \theta=r_{x}^{0} / R, \quad \sin \theta=r_{y}^{0} / R .
$$

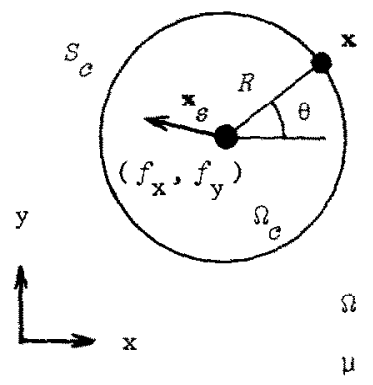

Fig. 1. Geometry of an infinitesimal sphere centered at $\boldsymbol{x}_{s}$. 
Then (8) and (9) can be written as

and

$$
\begin{aligned}
& u=A\left\{-f_{x}\left(\ln R+\sin ^{2} \theta\right)+f_{y} \sin \theta \cos \theta\right\}, \\
& u=A\left\{-f_{y}\left(\ln R+\cos ^{2} \theta\right)+f_{x} \sin \theta \cos \theta\right\}
\end{aligned}
$$

$$
p=C\left(f_{x} \cos \theta+f_{y} \sin \theta\right) / R \text {. }
$$

Let $\Omega_{\mathrm{c}}$ be an infinitesimal closed circle with radius $R$ around $\boldsymbol{x}_{\mathrm{s}}$, and let $S_{\mathrm{c}}$ be its surface. Then from $(5 a)$, we have

$$
\begin{aligned}
0 & =\int_{\Omega_{\mathrm{c}}}\left[\mu \nabla^{2} u(x)-\partial p(\boldsymbol{x}) / \partial x+f_{x} \delta\left(\boldsymbol{x}-\boldsymbol{x}_{s}\right)\right] \mathrm{d} \Omega \\
& =\int_{S_{\mathrm{c}}}[\mu\{(\partial u / \partial x) \cos \theta+(\partial u / \partial y) \sin \theta\}-p \cos \theta] \mathrm{d} S+f_{x} .
\end{aligned}
$$

Here the divergence theorem is applied. Noting $\mathrm{d} S=R \mathrm{~d} \theta$, (15) can be expressed as

$$
f_{x}=\int_{0}^{2 \pi}[p \cos \theta-\mu\{(\partial u / \partial x) \cos \theta+(\partial u / \partial y) \sin \theta\}] R \mathrm{~d} \theta=\pi f_{x}(C+2 \mu A) .
$$

Evidently $A$ and $C$ by (11) realize (16), and hence the velocity and pressure by (8) and (9) satisfy (5a) even at $x=x_{s}$. In the same way, we can prove that $(5 b)$ is always adequate.

REMARK 3.2. The whole-space problem is not well posed for a single body force. The natural boundary condition at infinity requires the force balance condition (7a) and (7b). Therefore, solutions of (8) and (9) should be linearly superposed for the balanced body forces.

THEOREM 3.3. For the whole-space problem, the stream function by (6) can be given as

$$
\psi=-A\left(f_{x} r_{y}^{0}-f_{y} r_{x}^{0}\right) \ln R .
$$

Here we neglect the integral constant.

REMARK 3.4. The incompressible viscous stream function of (17) is not harmonic, i.e. $\nabla^{2} \psi \neq 0$.

REMARK 3.5. The potential function $\phi$ (defined by $\partial \phi / \partial x=u, \partial \phi / \partial y=v$ and $\phi \perp \psi$ ) does not exist in the Stokes flow.

\section{Half-space problem}

We next consider the half-space $\Omega$. On the half-space boundary $\Gamma_{h}^{+}$of $y=h, v$ should be zero (see Fig. 2). The natural boundary condition at infinity should be applied also. 


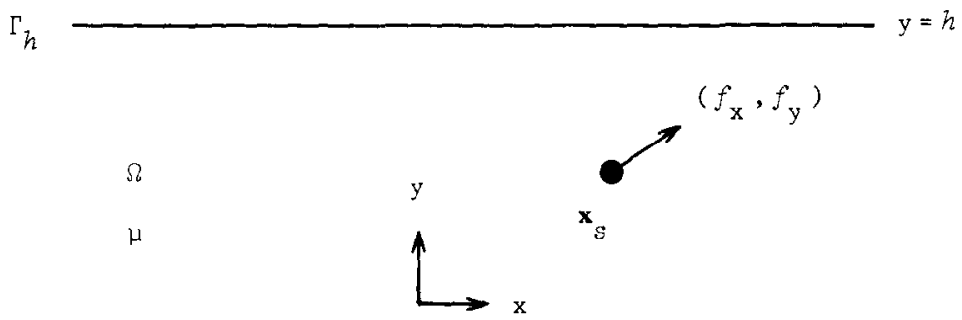

Fig. 2. Geometry of the half-space problem.

THEOREM 4.1. For the half-space problem, the velocity and pressure at $\boldsymbol{x}$ can be written explicitly as

$$
\begin{aligned}
& u=A\left[-f_{x} \ln R-r_{y}^{0}\left(f_{x} r_{y}^{0}-f_{y} r_{x}^{0}\right) / R^{2}-f_{x} \ln R_{0,1}-r_{y}^{1}\left(f_{x} r_{y}^{1}+f_{y} r_{x}^{0}\right) / R_{0,1}^{2} 1\right. \\
& v=A\left[-f_{y} \ln R-r_{x}^{0}\left(f_{y} r_{x}^{0}-f_{x} r_{y}^{0}\right) / R^{2}+f_{y} \ln R_{0,1}+r_{x}^{0}\left(f_{y} r_{x}^{0}+f_{x} r_{y}^{1}\right) / R_{0,1}^{2}\right]
\end{aligned}
$$

and

where

$$
p=C\left[\left(f_{x} r_{x}^{0}+f_{y} r_{y}^{0}\right) / R^{2}+\left(f_{x} r_{x}^{0}-f_{y} r_{y}^{1}\right) / R_{0,1}^{2}\right]
$$

$$
y_{s}^{1}=-y_{s}+2 h, \quad r_{y}^{1}=y-y_{s}^{1}, \quad R_{0,1}=\left\{\left(r_{x}^{0}\right)^{2}+\left(r_{y}^{1}\right)^{2}\right\}^{1 / 2} .
$$

PROOF. In whole-space, we place an image force $\left(f_{x},-f_{y}\right)$ at the image point $x_{s}^{1}=\left(x_{s}, y_{s}^{1}\right)$. Then we can solve the half-space problem as the whole-space one with the image body force. On the half-space boundary $\Gamma_{h}^{+}, v$ due to $f_{x}$ at $\boldsymbol{x}_{s}$ is cancelled with $v$ related to the image force $f_{x}$ at $\boldsymbol{x}_{s}^{1}$, while $v$ caused by $f_{y}$ at $\boldsymbol{x}_{s}$ is extinguished by $v$ associated with $-f_{y}$ at $\boldsymbol{x}_{s}^{1}$. Hence $v=0$ on $\Gamma_{h}^{+}$.

REMARK 4.2. In the half-space problem, the force balance condition is required only in the $x$-direction, since $f_{y}$ is balanced with its image force $-f_{y}$. Evidently $f_{y}$ should not be placed on $\Gamma_{h}^{+}$.

$R E M A R K$ 4.3. On $\Gamma_{h}^{+}$we have no tangential traction force, since $\partial u / \partial y=\partial v / \partial x=0$.

THEOREM 4.4. For the half-space problem, the stream function can be written as

$$
\psi=-A\left[\left(f_{x} r_{y}^{0}-f_{y} r_{x}^{0}\right) \ln R+\left(f_{x} r_{y}^{1}+f_{y} r_{x}^{0}\right) \ln R_{0.1}\right] .
$$

Here we neglect the integral constant.

\section{Infinite fissure problem}

The domain we next consider is the infinite fissure with two walls $\Gamma_{h}^{+}$of $y=h$ and $\Gamma_{h}^{-}$of $y=-h$ (see Fig. 3). On the walls, $v$ should be zero. The infinite fissure problem with the natural boundary condition at infinity can be solved by applying, successively, the image method. 


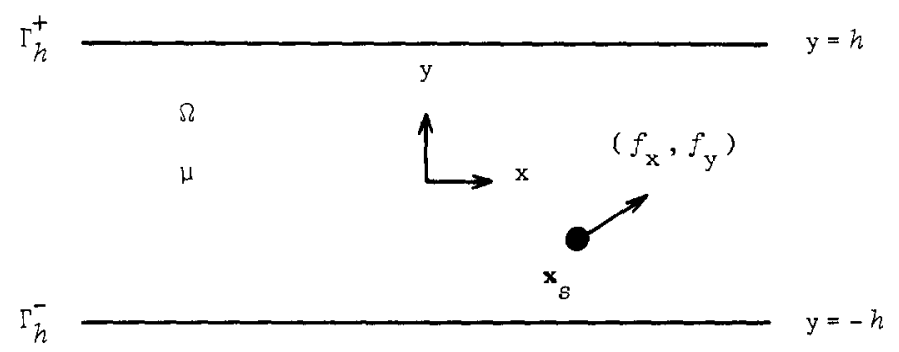

Fig. 3. Geometry of the infinite fissure problem.

THEOREM 5.1. For the infinite fissure problem, the velocity and pressure can be expressed as

$$
\begin{aligned}
& u=A \sum_{n=-\infty}^{\infty}\left[-f_{x} \ln R_{0, n}-r_{y}^{n}\left\{f_{x} r_{y}^{n}-(-1)^{n} f_{y} r_{x}^{0}\right\} / R_{0, n}^{2}\right], \\
& v=A \sum_{n=-\infty}^{\infty}\left[-(-1)^{n} f_{y} \ln R_{0, n}-r_{x}^{0}\left\{(-1)^{n} f_{y} r_{x}^{0}-f_{x} r_{y}^{n}\right\} / R_{0, n}^{2}\right]
\end{aligned}
$$

and

$$
p=C \sum_{n=-\infty}^{\infty}\left\{f_{x} r_{x}^{0}+(-1)^{n} f_{y} r_{y}^{n}\right\} / R_{0, n}^{2}
$$

Here

$$
y_{s}^{n}=(-1)^{n} y_{s}+2 n h, \quad r_{y}^{n}=y-y_{s}^{n}, \quad R_{0, n}=\left\{\left(r_{x}^{0}\right)^{2}+\left(r_{y}^{n}\right)^{2}\right\}^{1 / 2}, n=0, \pm 1, \ldots, \pm \infty .
$$

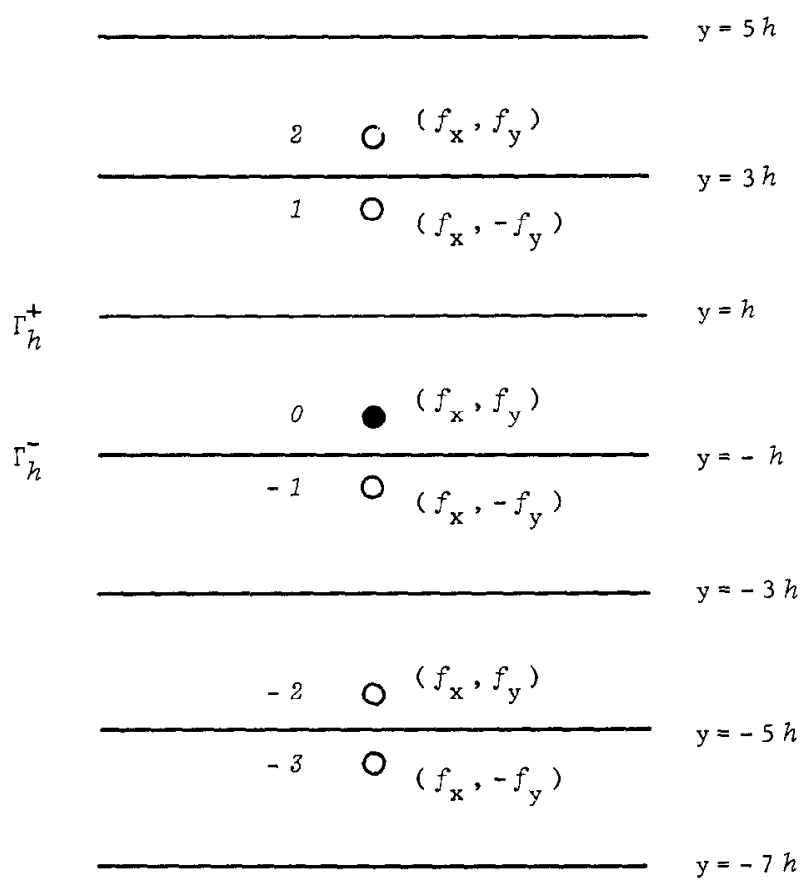

Fig. 4. Infinite series of image sources. 
PROOF. Let $\left(f_{x}, f_{y}\right)$ at $x_{s}$ correspond to $n=0$. With respect to $\Gamma_{h}^{+}$, its image force $\left(f_{x},-f_{y}\right)$ is placed at $\left(x_{s}, y_{s}^{1}\right)$ associated with $n=1$. Similarly with respect to $\Gamma_{h}^{-}$, we place the image force $\left(f_{x},-f_{y}\right)$ at $\left(x_{s}, y_{s}^{-1}\right)$ related to $n=-1$. These image procedures are successively applied such that the $(n+1)$ st force is the image of the $-n$th force with respect to $\Gamma_{h}^{+}$, while the $n$th force yields the $-(n+1)$ st one through $\Gamma_{\bar{h}}^{-}$(see Fig. 4).

Our infinite fissure problem can thus be solved as the whole-space one with an infinite series of image forces $\left(f_{x},(-1)^{n} f_{y}\right)$ at $\left(x_{s}, y_{s}^{n}\right)$.

REMARK 5.2. In the infinite fissure problem, only the force balance condition (7a) is needed just like in the previous half-space problem.

THEOREM 5.3. For the infinite fissure problem, the stream function can be written as

$$
\psi=-A \sum_{n=-\infty}^{\infty}\left\{f_{x} r_{y}^{n}-(-1)^{n} f_{y} r_{x}^{0}\right\} \ln R_{0, n} .
$$

Here the integral constant is neglected.

\section{Analytical solutions for the rectangle problem based on the image method}

We now consider the rectangular domain $\Omega$ with $\Gamma_{h}^{ \pm}$of $y= \pm h$ and $\Gamma_{\mathrm{B}}^{ \pm}$of $x= \pm g$ (Fig. 5). On $\Gamma_{h}^{ \pm} v$ should be zero, while $u$ should be zero on $\Gamma_{g}^{ \pm}$.

THEOREM 6.1. The analytical solutions for the rectangle problem can be given as

$$
\begin{aligned}
& u=A \sum_{m=-\infty}^{\infty} \sum_{n=-\infty}^{\infty}\left[-(-1)^{m} f_{x} \ln R_{m, n}-r_{y}^{n}\left\{(-1)^{m} f_{x} r_{y}^{n}-(-1)^{n} f_{y} r_{x}^{m}\right\} / R_{m, n}^{2}\right], \\
& v=A \sum_{m=-\infty}^{\infty} \sum_{n=-\infty}^{\infty}\left[-(-1)^{n} f_{y} \ln R_{m, n}-r_{x}^{m}\left\{(-1)^{n} f_{y} r_{x}^{m}-(-1)^{m} f_{x} r_{y}^{n}\right\} / R_{m, n}^{2}\right]
\end{aligned}
$$

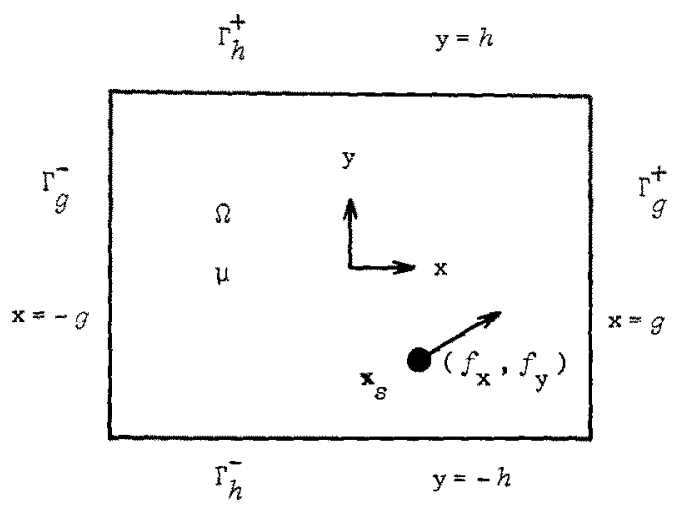

Fig. 5. Geometry of the rectangle problem. 


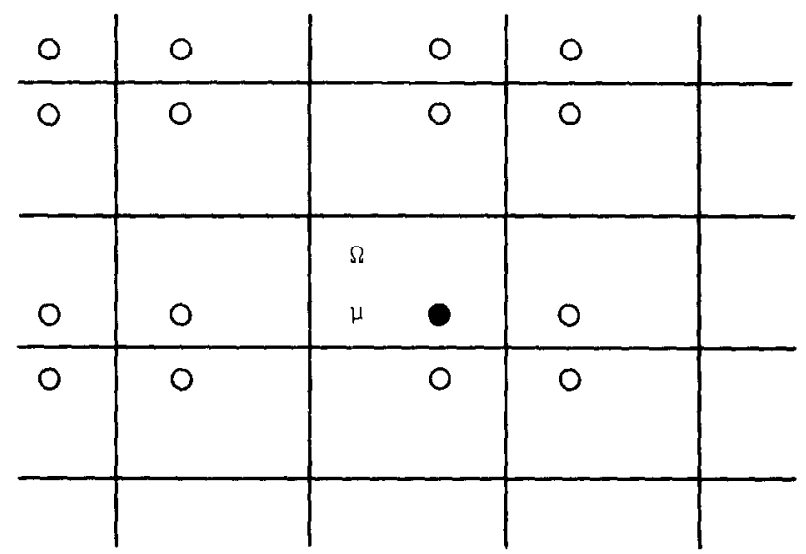

Fig. 6. Distributions of two-dimensional image sources.

and

$$
p=C \sum_{m=-\infty}^{\infty} \sum_{n=-\infty}^{\infty}\left[(-1)^{m} f_{x} r_{x}^{m}+(-1)^{n} f_{y} r_{y}^{n}\right] / R_{m, n}
$$

Here

$$
\begin{aligned}
& x_{s}^{m}=(-1)^{m} x_{s}+2 m g, \quad r_{x}^{m}=x-x_{s}^{m}, \quad R_{m, n}=\left\{\left(r_{x}^{m}\right)^{2}+\left(r_{y}^{n}\right)^{2}\right\}^{1 / 2} \\
& \quad m, n=0, \pm 1, \ldots, \pm \infty
\end{aligned}
$$

PROOF. To the infinite series of forces in the preceding fissure problem, we apply the image procedure with respect to $\Gamma_{g}^{ \pm}$(see Fig. 6). Our rectangle problem can then be solved as the whole-space one with $\left((-1)^{m} f_{x},(-1)^{n} f_{y}\right)$ at $\left(x_{s}^{m}, y_{s}^{n}\right)$.

REMARK 6.2. The incompressible rectangle problem is well posed even for a point force. It is evident, however, that $f_{x}$ should not be placed on $\Gamma_{\mathrm{g}}^{ \pm}$, while $f_{y}$ should not be on $\Gamma_{h}^{ \pm}$. For velocity constraints see Fig. 7.

THEOREM 6.3. For the rectangle problem, the stream function is given as

$$
\psi=-A \sum_{m=-\infty}^{\infty} \sum_{n=-\infty}^{\infty}\left[(-1)^{n} f_{x} r_{y}^{n}-(-1)^{m} f_{y} r_{x}^{m}\right] \ln R_{m, n}
$$

Here the integral constant is neglected.

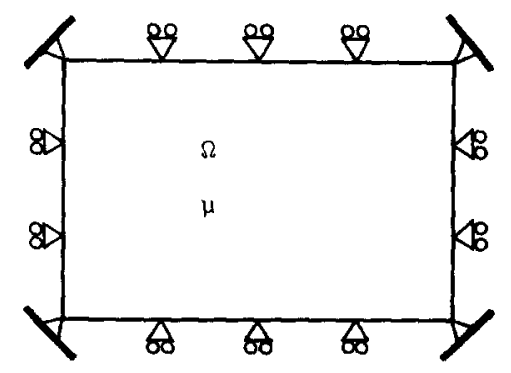

Fig. 7. Velocity constraints on the boundaries. 
REMARK 6.4. The velocity and pressure are unbounded naturally at the source point $\boldsymbol{x}_{s}$. The stream function, on the other hand, is bounded everywhere even at $\boldsymbol{x}=\boldsymbol{x}_{s}$.

Consider, for example, $r_{y}^{0} \ln R_{0,0}$. Then putting $r_{x}^{0}=\alpha t$ and $r_{y}^{0}=\beta t(t>0)$ at $x=x_{s}$, it extinguishes such that $\beta t \ln \left[t \sqrt{\alpha^{2}+\beta^{2}}\right] \rightarrow 0$ as $t \rightarrow 0^{+}$. Here $\alpha$ and $\beta$ denote appropriate constants.

\section{Some formulae related to the potential problem}

Analytical solutions for the steady Stokes flow within the rectangular domain are given in the form of infinite series with $m$ and $n$, which are not so preferable in computations. In this section, we present some formulae to simplify our solutions.

The problem we consider is the electrical infinite fissure $\Omega$ of unit specific conductivity between infinite spaces $\Omega^{+}$and $\Omega^{-}$of conductivity $\infty$ (Fig. 8). Let only a point source of unit intensity be placed at $\boldsymbol{x}_{s}^{m}=\left(x_{s}^{m}, y_{s}\right)$ with $\Omega$. Then the potential $\phi_{m}(\boldsymbol{x})$ at $\boldsymbol{x}$ in $\Omega$ is governed by

$$
\nabla^{2} \phi_{m}(x)+\delta\left(x-x_{s}^{m}\right)=0 .
$$

The boundary conditions on $\Gamma_{h}^{ \pm}$of $y= \pm h$ are

$$
\phi_{m}(x)=0, \quad x \in \Gamma_{h}^{ \pm} .
$$

LEMMA 7.1. For the electrical fissure problem, the potential at $\boldsymbol{x}$ in $\Omega$ can be written as

$$
4 \pi \phi_{m}(x)=-\ln \left[F^{-}\left(x, y, x_{s}^{m}, y_{s}, h\right) / F^{+}\left(x, y, x_{s}^{m}, y_{s}, h\right)\right]
$$

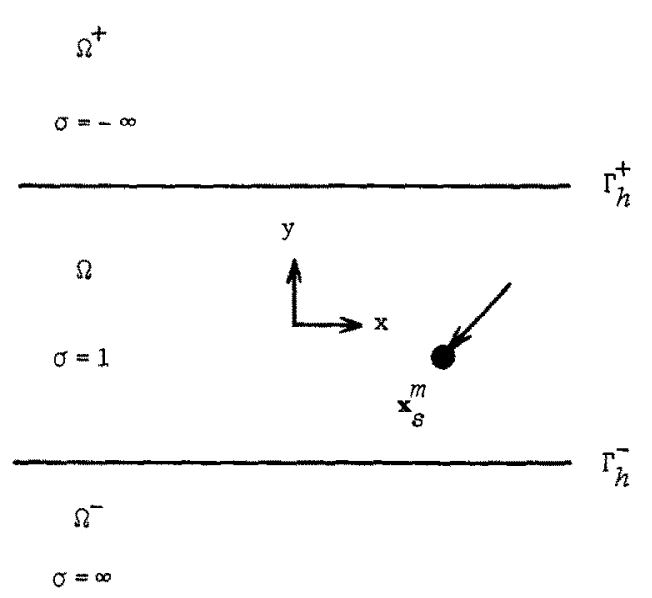

Fig. 8. Geometry of the electrical infinite fissure problem. An infinite fissure is embedded in otherwise perfectly conductive whole-space.
Fig. 9. Transformed space after the conformal mapping of (34). 
where

$$
F^{ \pm}\left(x, y, x_{s}^{m}, y_{s}, h\right)=\mathrm{e}^{\pi x / h}+\mathrm{e}^{\pi x_{s}^{m} / h} \pm 2 \mathrm{e}^{\pi\left(x+x_{s}^{m}\right) / 2 h} \cos \pi\left(y \pm y_{s}\right) / 2 h
$$

PROOF. Under the conformal mapping of the form

$$
\pi(x+\mathrm{i} y)=2 h \ln (X+\mathrm{i} Y),
$$

the infinite fissure of Fig. 8 can conformally be translated into the half-space of Fig. 9. Here i designates the imaginary unit. For further details, see $[14,15]$.

LEMMA 7.2. For the electrical fissure problem, the image procedure yields the solutions in another form of

$$
2 \pi \phi_{m}(x)=-\sum_{n=-\infty}^{\infty}(-1)^{n} \ln R_{m, n} .
$$

REMARK 7.3. The solution of (32) or (35) is adequate only after the linear superposition for balanced current sources just like in the preceding Stokes flow within the infinite fissure.

Lemmas 7.1 and 7.2 thus produce the following theorems, which are applicable to the steady Stokes flow.

THEOREM 7.4. Let us define the $V_{m}$ function by

$$
V_{m}=\frac{1}{2} \ln \left[F^{-}\left(x, y, x_{s}^{m}, y_{s}, h\right) / F^{+}\left(x, y, x_{s}^{m}, y_{s}, h\right)\right] .
$$

Then we have

$$
V_{m}=\sum_{n=-\infty}^{\infty}(-1)^{n} \ln R_{m, n}+D_{m},
$$

where $D_{m}$ designates an appropriate constant.

THEOREM 7.5. Let us define the $U_{n}$ function by

$$
U_{n}=\frac{1}{2} \ln \left[F^{-}\left(y, x, y_{s}^{n}, x_{s}, g\right) / F^{+}\left(y, x, y_{s}^{n}, x_{s}, g\right)\right] .
$$

Then we have

$$
U_{n}=\sum_{m=-\infty}^{\infty}(-1)^{m} \ln R_{m, n}+D_{n}
$$

where $D_{n}$ designates an appropriate constant. 
THEOREM 7.6. The following relations hold:

$$
\begin{aligned}
\partial V_{m} / \partial y & =\frac{\pi}{2 h} \mathrm{e}^{\pi\left(x+x_{s}^{m}\right) / 2 h}\left[\frac{\sin \pi\left(y-y_{s}\right) / 2 h}{F^{-}\left(x, y, x_{s}^{m}, y_{s}, h\right)}+\frac{\sin \pi\left(y+y_{s}\right) / 2 h}{F^{+}\left(x, y, x_{s}^{m}, y_{s}, h\right)}\right] \\
& =\sum_{n=-\infty}^{\infty}(-1)^{n} r_{y}^{n} / R_{m, n}^{2}, \\
\partial U_{n} / \partial x & =\frac{\pi}{2 g} \mathrm{e}^{\pi\left(y+y_{s}^{n}\right) / 2 g}\left[\frac{\sin \pi\left(x-x_{s}\right) / 2 g}{F^{-}\left(y, x, y_{s}^{n}, x_{s}, g\right)}+\frac{\sin \pi\left(x+x_{s}\right) / 2 g}{F^{+}\left(y, x, y_{s}^{n}, x_{s}, g\right)}\right] \\
& =\sum_{m=-\infty}^{\infty}(-1)^{m} r_{x}^{m} / R_{m, n}^{2}, \\
\partial V_{m} / \partial x & =\frac{\pi}{2 h}\left[\frac{\mathrm{e}^{\pi x / h}-\mathrm{e}^{\pi\left(x+x_{s}^{m}\right) / 2 h} \cos \pi\left(y-y_{s}\right) / 2 h}{F^{-}\left(x, y, x_{s}^{m}, y_{s}, h\right)}-\frac{\mathrm{e}^{\pi x / h}+\mathrm{e}^{\pi\left(x+x_{s}^{m}\right) / 2 h} \cos \pi\left(y+y_{s}\right) / 2 h}{F^{+}\left(x, y, x_{s}^{m}, y_{s}, h\right)}\right] \\
& =r_{x}^{m} \sum_{n=-\infty}^{\infty}(-1)^{n} / R_{m, n}^{2}, \\
\partial U_{n} / \partial y & =\frac{\pi}{2 g}\left[\frac{\mathrm{e}^{\pi y / g}-\mathrm{e}^{\pi\left(y+y_{s}^{n}\right) / 2 g} \cos \pi\left(x-x_{s}\right) / 2 g}{F^{-}\left(y, x, y_{s}^{n}, x_{s}, g\right)}-\frac{\mathrm{e}^{\pi y / g}+\mathrm{e}^{\pi\left(y+y_{s}^{n}\right) / 2 g} \cos \pi\left(x+x_{s}\right) / 2 g}{F^{+}\left(y, x, y_{s}^{n}, x_{s}, g\right)}\right] \\
& =r_{y}^{n} \sum_{m=-\infty}^{\infty}(-1)^{m} / R_{m, n}^{2} .
\end{aligned}
$$

\section{Simplified solutions for the rectangle problem}

We can now simplify our solutions in Theorems 6.1 and 6.3 by using the preceding formulas. It is especially noted that the simplified solutions are given also in the form of infinite series, but only the one-directional expansion is needed.

THEOREM 8.1. For the rectangle problem, the incompressible viscous velocity and pressure can be written simply as

$$
\begin{aligned}
& u=A\left[-f_{x} \sum_{n=-\infty}^{\infty}\left\{U_{n}+r_{y}^{n}\left(\partial U_{n} / \partial y\right)\right\}+f_{y} \sum_{m=-\infty}^{\infty} r_{x}^{m}\left(\partial V_{m} / \partial y\right)\right] \\
& v=A\left[-f_{y} \sum_{m=-\infty}^{\infty}\left\{V_{m}+r_{x}^{m}\left(\partial V_{m} / \partial x\right)\right\}+f_{x} \sum_{n=-\infty}^{\infty} r_{y}^{n}\left(\partial U_{n} / \partial x\right)\right]
\end{aligned}
$$

and

$$
p=C\left[f_{x} \sum_{n=-\infty}^{\infty}\left(\partial U_{n} / \partial x\right)+f_{y} \sum_{m=-\infty}^{\infty}\left(\partial V_{m} / \partial y\right)\right] \text {. }
$$


THEOREM 8.2. For the rectangle problem, the incompressible viscous stream function can be written simply as

$$
\psi=-A\left[f_{x} \sum_{n=-\infty}^{\infty} r_{y}^{n} U_{n}-f_{y} \sum_{m=-\infty}^{\infty} r_{x}^{m} V_{m}\right]
$$

Here we neglect the integral constant.

We then deal with the stress-strain relation for the incompressible viscous fluid.

THEOREM 8.3. For the rectangle problem, stress components $\sigma_{x}, \sigma_{y}$ and $\tau_{x y}$ are given by

$$
\begin{aligned}
\sigma_{x}= & C\left[-f_{x} \sum_{n=-\infty}^{\infty}\left\{2\left(\partial U_{n} / \partial x\right)+r_{y}^{n}\left(\partial^{2} U_{n} / \partial x \partial y\right)\right\}+f_{y} \sum_{m=-\infty}^{\infty} r_{x}^{m}\left(\partial^{2} V_{m} / \partial x \partial y\right)\right], \\
\sigma_{y}= & C\left[-f_{y} \sum_{m=-\infty}^{\infty}\left\{2\left(\partial V_{m} / \partial y\right)+r_{x}^{m}\left(\partial^{2} V_{m} / \partial x \partial y\right)\right\}+f_{x} \sum_{n=-\infty}^{\infty} r_{y}^{n}\left(\partial^{2} U_{n} / \partial x \partial y\right)\right], \\
\tau_{x y}= & C\left[f_{x} \sum_{n=-\infty}^{\infty}\left\{r_{y}^{n}\left(\partial^{2} U_{n} / \partial x^{2}\right)-\partial U_{n} / \partial y\right\}+f_{y} \sum_{m=-\infty}^{\infty}\left\{r_{x}^{m}\left(\partial^{2} V_{m} / \partial y^{2}\right)-\partial V_{m} / \partial x\right\}\right. \\
& \left.-\pi\left(f_{x}+f_{y}\right) \delta\left(x-x_{s}\right)\right] .
\end{aligned}
$$

PROOF. Strain-rate components $\varepsilon_{x}, \varepsilon_{y}$ and $\gamma_{x y}$ are defined by

$$
\varepsilon_{x}=\partial u / \partial x, \quad \varepsilon_{y}=\partial v / \partial y, \quad 2 \gamma_{x y}=\partial u / \partial y+\partial v / \partial x .
$$

Stress components can then be written as

$$
\sigma_{x}=2 \mu \varepsilon_{x}-p, \quad \sigma_{y}=2 \mu \varepsilon_{y}-p, \quad \tau_{x y}=2 \mu \gamma_{x y} .
$$

Substitution of (44) and (45) into (48) and (49) thus completes the proof.

REMARK 8.4. In deriving $\tau_{x y}$, notice that

$$
\nabla^{2} V_{0}-2 \pi \delta\left(x-x_{s}\right)=0 .
$$

Evidently $V_{m}(m \neq 0)$ as well as $U_{n}(n \neq 0)$ are harmonic.

REMARK 8.5. Let the outward normal be expressed as $\left(n_{x}, n_{y}\right)$ on the boundaries. Then the traction force $\left(t_{x}, t_{y}\right)$ can be obtained as

$$
t_{x}=n_{x} \sigma_{x}+n_{y} \tau_{x y}, \quad t_{y}=n_{x} \tau_{x y}+n_{y} \sigma_{y} .
$$

In our rectangle problem, no tangential forces exist. 


\section{Examinations of boundary element solutions}

We can now examine currently used numerical techniques such as the finite element and boundary element methods. Examples we present are associated with a rectangle of $g=4$ and $h=2$ with a homogeneous viscosity of $\mu=1 / 2 \pi$ (Fig. 10). Body forces of $\left(\frac{1}{2} \sqrt{3}, \frac{1}{2}\right)$ and $\left(-\frac{1}{2} \sqrt{3},-\frac{1}{2}\right)$ are applied at source points $(2,1)$ and $(2,-1)$, respectively. Our computations are made by using finite series of $\sum_{-N}^{N}$ in place of infinite ones of $\Sigma_{-\infty}^{\infty}$. Here $N$ is increased until the sufficient convergence is attained.

Table 1 shows the convergence of the velocity $u$, pressure $p$ and stress $\sigma_{x}$ at the origin $(0,0)$. All values converge quite rapidly, while larger $N$ is needed in computing the stream function as shown in Table 2 . At nodes regularly placed on a grid without vacancy, computed results are listed in Tables 3 to 5 .

We then apply the boundary element method (see Appendices A and B) associated with the whole-space fundamental solutions to this rectangle problem. The boundary surface is divided into 24 boundary elements of length 1 , on which the piecewise linear approximations are made

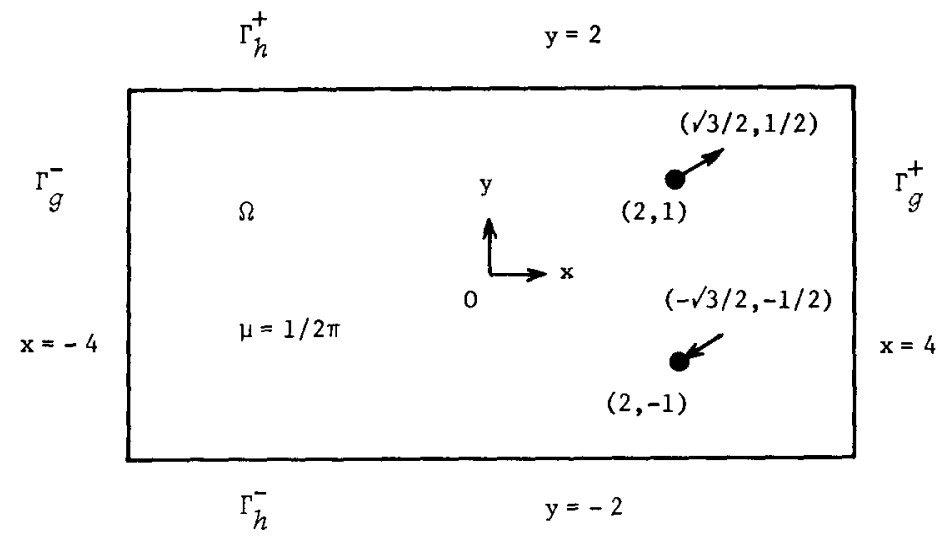

Fig. 10. Geometry of the example problem of $g=4$ and $h=2$. Body forces of intensity $\left(\frac{1}{2} \sqrt{3}, \frac{1}{2}\right)$ and $\left(-\frac{1}{2} \sqrt{3},-\frac{1}{2}\right)$ are applied at the source points of $(2,1)$ and $(2,-1)$, respectively. The isotropic and uniform viscosity of $\mu=1 / 2 \pi$ is assumed.

Table 1

Numerical solutions at the origin $(0,0)$ under the finite approximations. The infinite series of $\sum_{-\infty}^{\infty}$ is approximated by the finite one of $\Sigma_{-N}^{N}$

\begin{tabular}{cccc}
\hline$N$ & $u\left(\times 10^{-8}\right)$ & $p\left(\times 10^{-8}\right)$ & $\sigma_{x}\left(\times 10^{-8}\right)$ \\
\hline 0 & 6775377 & -1078335 & 3375079 \\
1 & 6813287 & -1080356 & 3394133 \\
2 & 6813287 & -1080356 & 3394133 \\
3 & 6813287 & -1080356 & 3394133 \\
\hline
\end{tabular}

Table 2

Computed stream function values under the finite approximations at the origin $(0,0)$

\begin{tabular}{llrl}
\hline$N$ & $\psi\left(\times 10^{-7}\right)$ & $N$ & $\psi\left(\times 10^{-7}\right)$ \\
\hline 0 & -6803068 & 8 & -7569628 \\
1 & -9199912 & 9 & -7569866 \\
2 & -6936128 & 10 & -7569811 \\
3 & -7762598 & 11 & -7569823 \\
4 & -7517060 & 12 & -7569820 \\
5 & -7583426 & 13 & -7569821 \\
6 & -7566445 & 14 & -7569821 \\
7 & -7570637 & 15 & -7569821 \\
\hline
\end{tabular}


Table 3

Solutions of the stream function and velocities in the rectangle problem

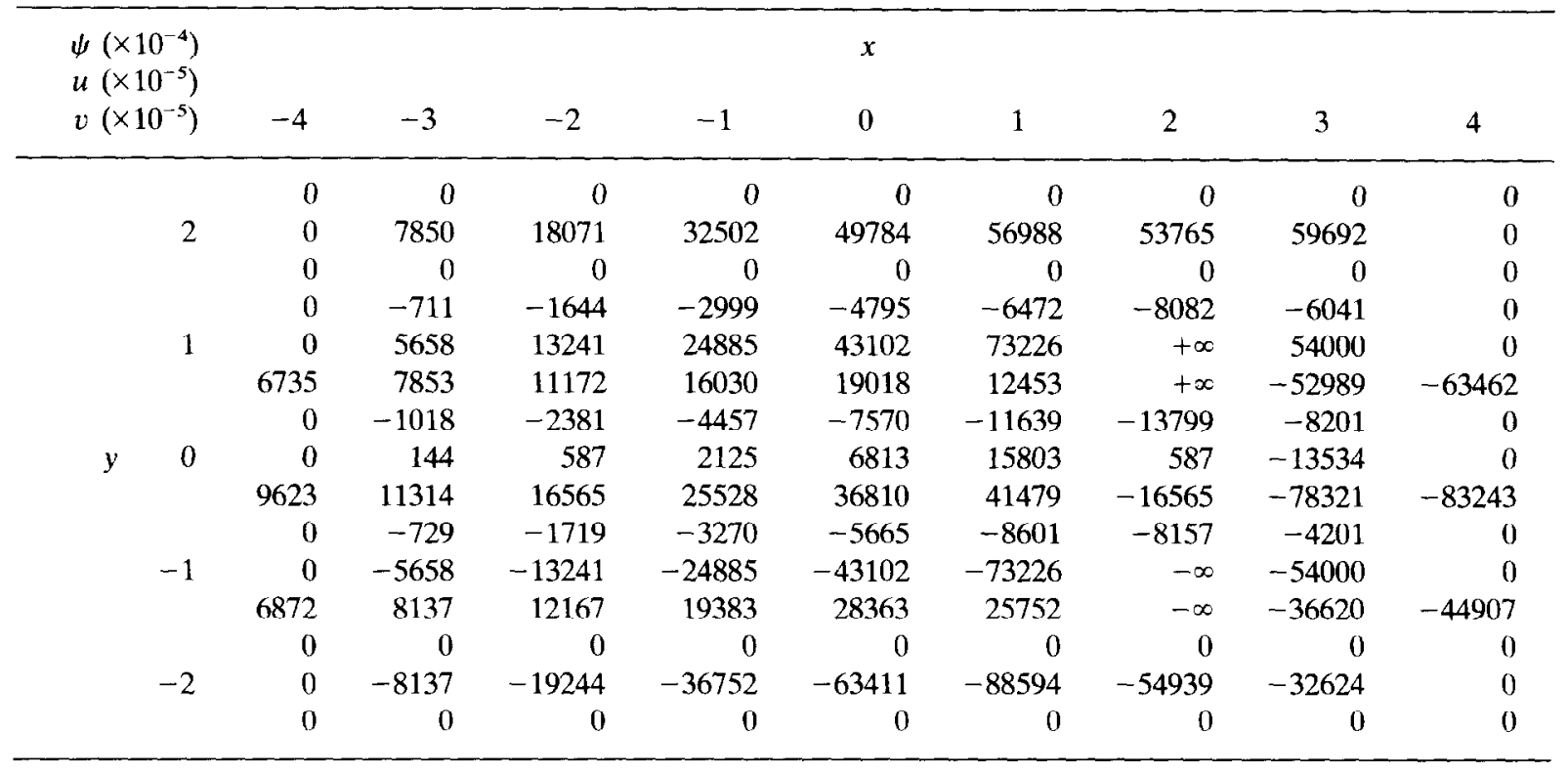

Table 4

Solutions of the pressure and strain components in the rectangle problem

\begin{tabular}{|c|c|c|c|c|c|c|c|c|c|c|}
\hline \multicolumn{2}{|c|}{$\begin{array}{r}p\left(\times 10^{-5}\right) \\
\varepsilon_{x}\left(\times 10^{-5}\right) \\
\gamma_{x y}\left(\times 10^{-5}\right)\end{array}$} & \multicolumn{9}{|c|}{$x$} \\
\hline \multirow{4}{*}{\multicolumn{2}{|c|}{2}} & -522 & -687 & -1271 & -2550 & -4735 & -5011 & 13865 & 18681 & 14313 \\
\hline & & 7449 & 8651 & 12131 & 16639 & 16000 & -6306 & 25582 & -38460 & -68524 \\
\hline & & 0 & 0 & 0 & 0 & 0 & 0 & 0 & 0 & 0 \\
\hline & & -372 & -493 & -936 & -2000 & -4518 & -12039 & $\pm \infty$ & 14532 & 9407 \\
\hline \multirow{3}{*}{\multicolumn{2}{|c|}{1}} & 5345 & 6287 & 9234 & 14458 & 22626 & 41718 & $\pm \infty$ & -62463 & -50597 \\
\hline & & 0 & 3223 & 6863 & 10239 & 9078 & -4500 & $\pm \infty$ & 7961 & 0 \\
\hline & & -4 & -10 & -47 & -225 & -1080 & -4991 & -12547 & -5206 & -2157 \\
\hline \multirow[t]{8}{*}{$y$} & \multirow[t]{3}{*}{0} & 107 & 223 & 781 & 2632 & 7269 & 7106 & -38495 & 9515 & 14431 \\
\hline & & 0 & 4853 & 10948 & 19343 & 30824 & 39414 & -13197 & 24924 & 0 \\
\hline & & 372 & 493 & 936 & 2000 & 4518 & 12039 & $\pm \infty$ & -14532 & -9407 \\
\hline & \multirow[t]{3}{*}{-1} & -5345 & -6287 & -9234 & -14458 & -22626 & -41718 & $\pm \infty$ & 62463 & 50597 \\
\hline & & 0 & 3616 & 8413 & 15504 & 23829 & 20241 & $\pm \infty$ & -11122 & 0 \\
\hline & & 530 & 707 & 1365 & 3000 & 6895 & 14994 & 11228 & -8268 & -10000 \\
\hline & \multirow[t]{2}{*}{-2} & -7663 & -9097 & -13694 & -21904 & -30538 & -7905 & 51408 & 19430 & 39662 \\
\hline & & 0 & 0 & 0 & 0 & 0 & 0 & 0 & 0 & 0 \\
\hline
\end{tabular}


Table 5

Solutions of the stress components in the rectangle problem.

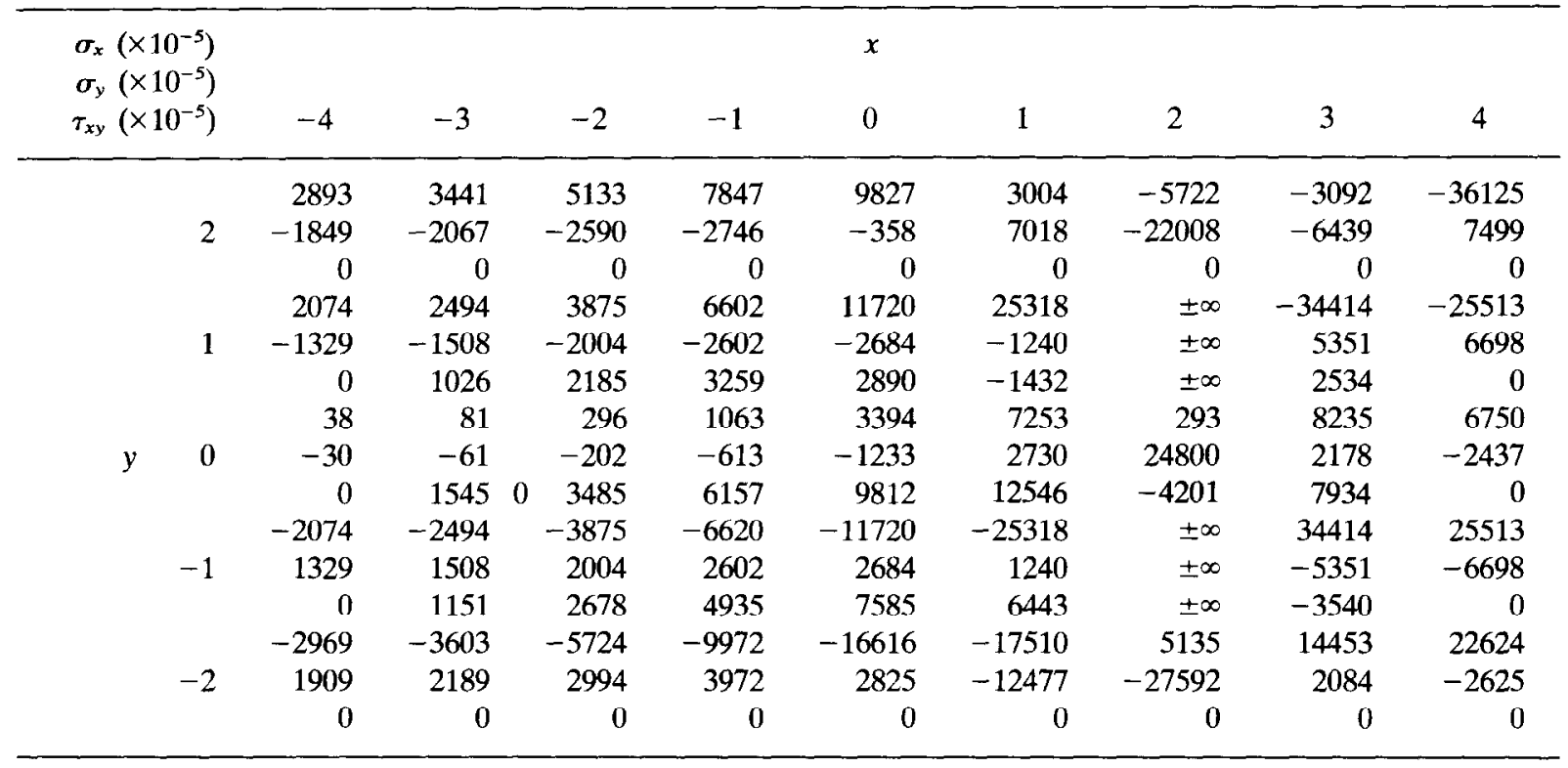

for the tangential velocity with the piecewise constant normal traction forces. Under the tangential-free boundary conditions that we are concerned with, we must determine 20 velocities at nodes on smooth boundaries and 24 traction forces on the boundary elements. For the tangential components nodes on smooth boundaries are chosen as $\boldsymbol{x}_{m}$, while centers of gravity of every boundary elements are selected as $\boldsymbol{x}_{\boldsymbol{l}}$ for the normal components.

Computed velocities on the boundaries are plotted in Figs. 11 and 12 together with the exact profiles. We also show the velocity and strain components along the line of $y=0$ in Figs. 13 and 14, respectively. Extremely accurate results are thus obtained.

Computed traction forces on $\Gamma_{h}^{+}$are drawn in Fig. 15, which are in good agreement with exact solutions. But the superconvergence like in the velocities cannot be attained. We then compute the pressure, and the results along the line of $y=0$ are plotted in Fig. 16. It is thus obvious that the accuracy of boundary element stress components is strongly influenced by that of the pressure.

\section{Concluding remarks}

We have developed analytical solutions of the steady Stokes flow in a rectangular domain as well as in an infinite fissure, which are applicable to the boundary integral techniques as typical fundamental solutions. Alternative expressions by the use of Fourier series have been already developed also, but they converge very slowly. It is emphasized that the quite rapid convergence inherent to our solutions is of great importance in the boundary element applications. 

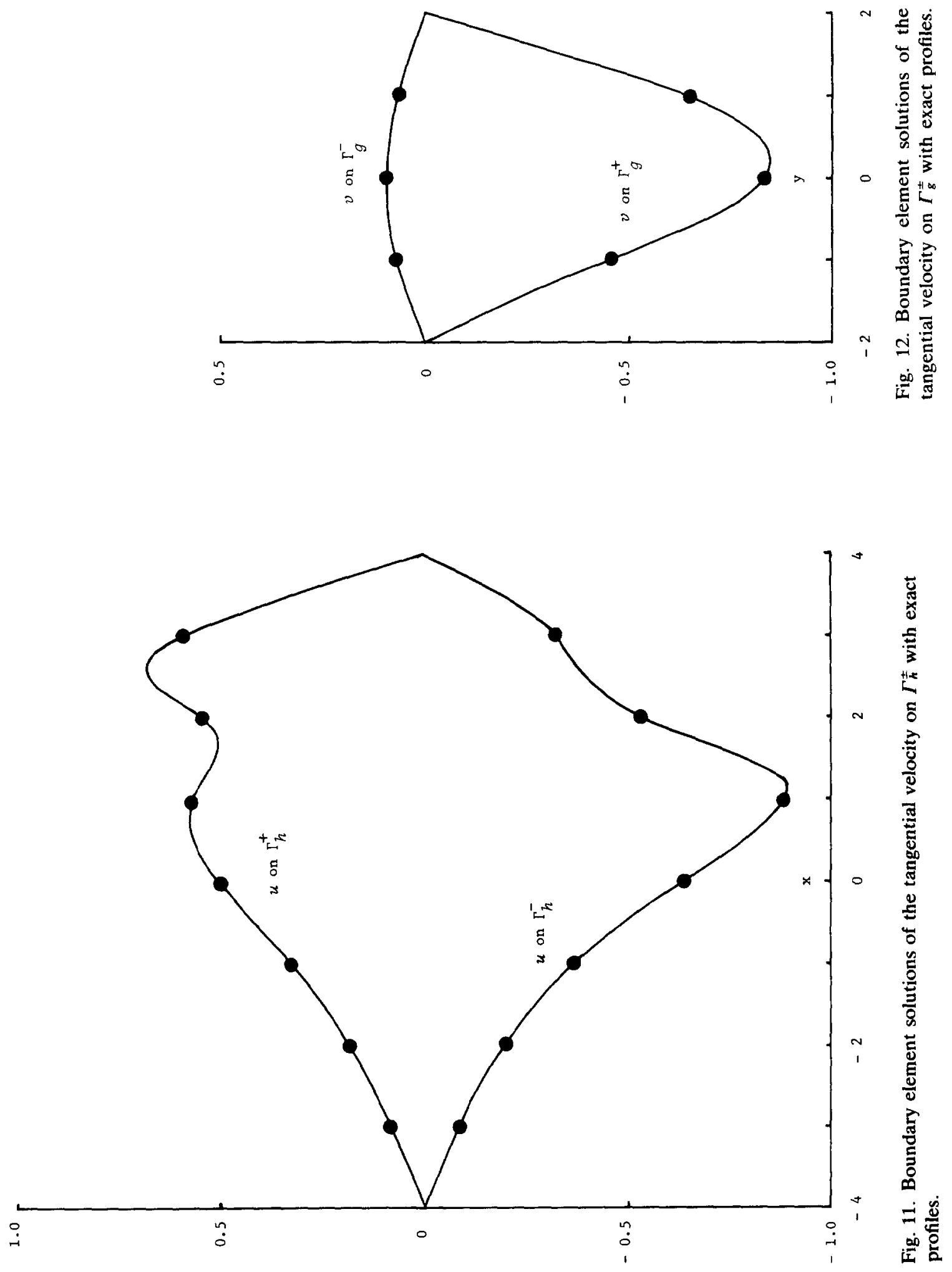


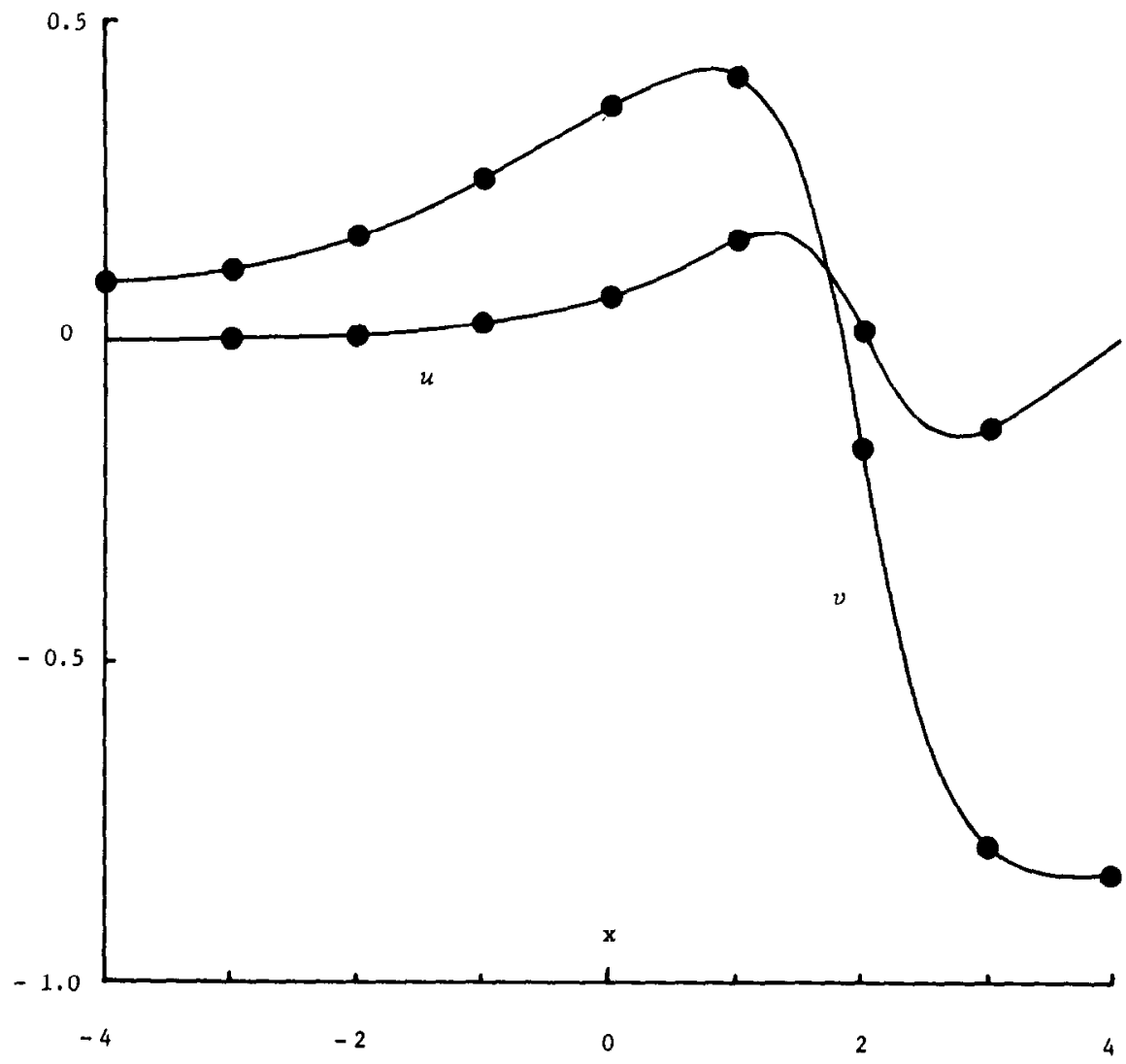

Fig. 13. Boundary element solutions of velocities along the line of $y=0$.

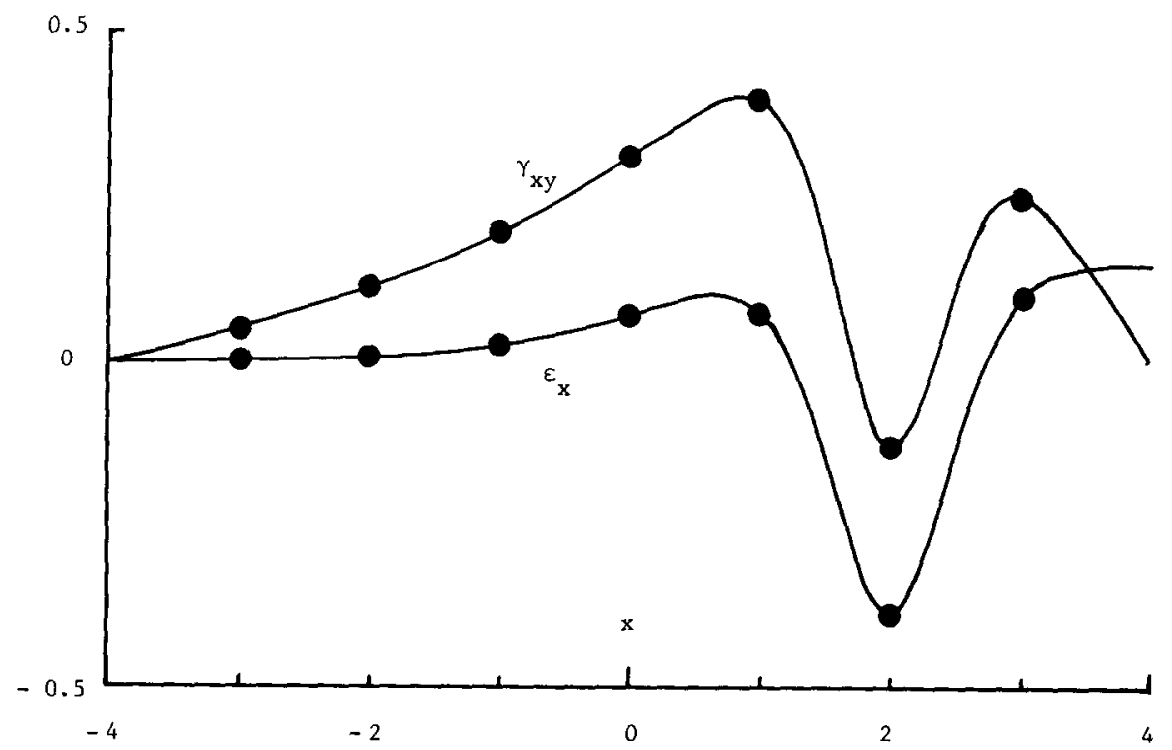

Fig. 14. Boundary element solutions of strain components along $y=0$. 

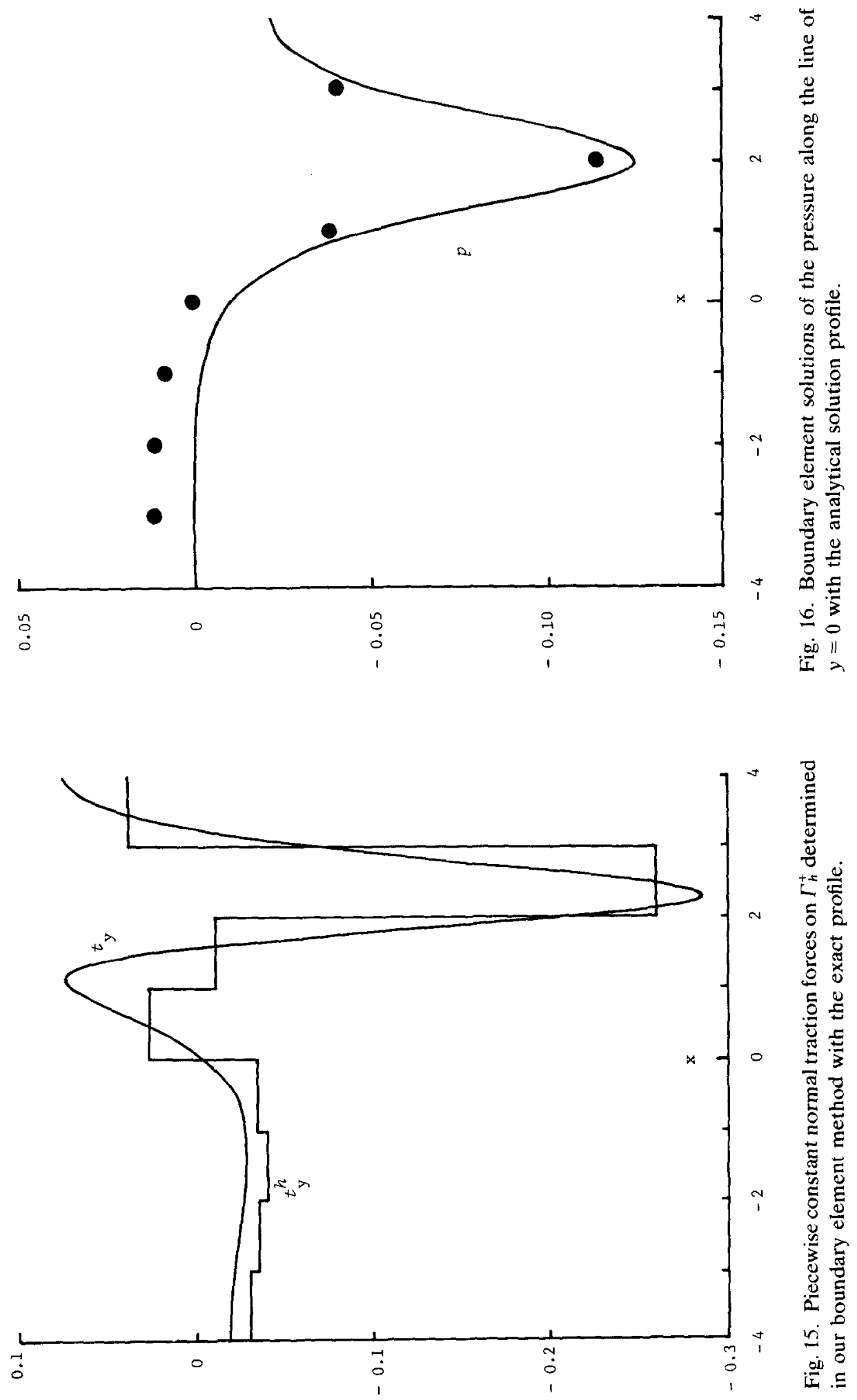


\section{Appendix A. Boundary integral equations associated with the whole-space fundamental solutions}

Referring to the whole-space solutions in (8) and (9), we introduce the fundamental solutions $\hat{\boldsymbol{u}}=(\hat{\boldsymbol{u}}, \hat{\boldsymbol{v}})$ and $\hat{p}$ by

$$
\begin{aligned}
& \hat{u}(\tilde{\boldsymbol{x}}, \boldsymbol{x})=\hat{u}^{1} \delta_{1 i}+\hat{u}^{2} \delta_{2 i}, \\
& \hat{v}(\tilde{\boldsymbol{x}}, \boldsymbol{x})=\hat{v}^{1} \delta_{1 i}+\hat{v}^{2} \delta_{2 i}, \\
& \hat{p}(\tilde{\boldsymbol{x}}, \boldsymbol{x})=\hat{p}^{1} \delta_{1 i}+\hat{p}^{2} \delta_{2 i} .
\end{aligned}
$$

Here $\delta_{i j}$ is the Kronecker delta $(i, j=1,2)$ and

$$
\begin{aligned}
& 4 \pi \mu \hat{u}^{1}=-\ln r-(\tilde{y}-y)^{2} / r^{2}, \\
& 4 \pi \mu \hat{v}^{1}=(\tilde{x}-x)(\tilde{y}-y) / r^{2}, \\
& 2 \pi \hat{p}^{1}=(\tilde{x}-x) / r^{2}, \\
& 4 \pi \mu \hat{u}^{2}=(\tilde{x}-x)(\tilde{y}-y) / r^{2}, \\
& 4 \pi \mu \hat{v}^{2}=-\ln r-(\tilde{x}-x)^{2} / r^{2}, \\
& 2 \pi \hat{p}^{2}=(\tilde{y}-y) / r^{2}
\end{aligned}
$$

where $r$ denotes the distance between $\boldsymbol{x}$ and $\tilde{\boldsymbol{x}}=(\tilde{\boldsymbol{x}}, \tilde{y})$ by

$$
r=\left[(\tilde{x}-x)^{2}+(\tilde{y}-y)^{2}\right]^{1 / 2} .
$$

Then the governing equations for the fundamental solutions can be written as

$$
\begin{aligned}
& \tilde{L}_{u}[\hat{\boldsymbol{u}}(\tilde{\boldsymbol{x}}, \boldsymbol{x})]+\lambda(\boldsymbol{x}) \delta_{1 i} \delta(\tilde{\boldsymbol{x}}-\boldsymbol{x})=0, \\
& \tilde{L}_{v}[\hat{\boldsymbol{u}}(\tilde{\boldsymbol{x}}, \boldsymbol{x})]+\lambda(\boldsymbol{x}) \delta_{2 i} \delta(\tilde{\boldsymbol{x}}-\boldsymbol{x})=0,
\end{aligned}
$$

Here the differential operators $\tilde{L}_{u}$ and $\tilde{L}_{v}$ are given by using the Laplacian $\tilde{\nabla}^{2}$ with respect to $\tilde{\boldsymbol{x}}$ such that

$$
\begin{aligned}
& \tilde{L}_{u}[\hat{\boldsymbol{u}}(\tilde{\boldsymbol{x}}, \boldsymbol{x})]=\mu \tilde{\nabla}^{2} \hat{u}(\tilde{\boldsymbol{x}}, \boldsymbol{x})-\partial \hat{p}(\tilde{\boldsymbol{x}}, \boldsymbol{x}) / \partial \tilde{\boldsymbol{x}}, \\
& \tilde{L}_{v}[\hat{\boldsymbol{u}}(\tilde{\boldsymbol{x}}, \boldsymbol{x})]=\mu \tilde{\nabla}^{2} \hat{v}(\tilde{\boldsymbol{x}}, \boldsymbol{x})-\partial \hat{p}(\tilde{\boldsymbol{x}}, \boldsymbol{x}) / \partial \tilde{y},
\end{aligned}
$$

and $\lambda(x)$ designates the positioning constant which can be geometrically defined [16]. form

Noting the governing equations (5) and (A.5), we adopt the weighted residual scheme of the

$$
\begin{aligned}
0= & \int_{\Omega} \hat{u}(\tilde{\boldsymbol{x}}, \boldsymbol{x})\left\{\tilde{L}_{u}[\boldsymbol{u}(\tilde{\boldsymbol{x}})]+f_{x} \delta\left(\tilde{\boldsymbol{x}}-\boldsymbol{x}_{s}\right)\right\} \mathrm{d} \tilde{\Omega}+\int_{\Omega} \hat{v}(\tilde{\boldsymbol{x}}, \boldsymbol{x})\left\{\tilde{L_{v}}[\boldsymbol{u}(\tilde{\boldsymbol{x}})]+f_{y} \delta\left(\tilde{\boldsymbol{x}}-\boldsymbol{x}_{s}\right)\right\} \mathrm{d} \tilde{\Omega} \\
& -\int_{\Omega} u(\tilde{\boldsymbol{x}})\left\{\tilde{L_{u}}[\hat{\boldsymbol{u}}(\tilde{\boldsymbol{x}}, \boldsymbol{x})]+\lambda(\boldsymbol{x}) \delta_{1 i} \delta(\tilde{\boldsymbol{x}}-\boldsymbol{x})\right\} \mathrm{d} \tilde{\Omega} \\
& -\int_{\Omega} v(\tilde{\boldsymbol{x}})\left\{\tilde{L_{v}}[\hat{\boldsymbol{u}}(\tilde{\boldsymbol{x}}, \boldsymbol{x})]+\lambda(\boldsymbol{x}) \delta_{2 i} \delta(\tilde{\boldsymbol{x}}-\boldsymbol{x})\right\} \mathrm{d} \tilde{\Omega} .
\end{aligned}
$$


Here the integrations and differentiations are taken with respect to $\tilde{\boldsymbol{x}}$. Applying the Green's theorem, (A.7) can be written as

$$
\begin{aligned}
\lambda(\boldsymbol{x})\left\{\delta_{1 i} u(\boldsymbol{x})+\delta_{2 i} v(\boldsymbol{x})\right\}= & \hat{u}\left(\boldsymbol{x}_{s}, \boldsymbol{x}\right) f_{x}+\hat{v}\left(\boldsymbol{x}_{s}, \boldsymbol{x}\right) f_{y}+\int_{\Gamma}\left\{t_{x}(\tilde{\boldsymbol{x}}) \hat{u}(\tilde{\boldsymbol{x}}, \boldsymbol{x})+t_{y}(\tilde{\boldsymbol{x}}) \hat{v}(\tilde{\boldsymbol{x}}, \boldsymbol{x})\right\} \mathrm{d} \tilde{\Gamma} \\
& -\int_{\Gamma}\left\{u(\tilde{\boldsymbol{x}}) \hat{t}_{\boldsymbol{x}}(\tilde{\boldsymbol{x}}, \boldsymbol{x})+v(\tilde{\boldsymbol{x}}) \hat{t}_{y}(\tilde{\boldsymbol{x}}, \boldsymbol{x})\right\} \mathrm{d} \tilde{\Gamma}
\end{aligned}
$$

Here the integrations are taken over the boundary $\Gamma$ with respect to $\tilde{x}$, and $\hat{t}_{x}$ and $\hat{t}_{y}$ are given by

$$
\begin{aligned}
& \hat{t}_{x}(\tilde{\boldsymbol{x}}, \boldsymbol{x})=-2(\tilde{\boldsymbol{x}}-x) \tilde{\boldsymbol{n}} \cdot(\tilde{\boldsymbol{x}}-\boldsymbol{x}) \hat{p}(\tilde{\boldsymbol{x}}, \boldsymbol{x}) / r^{2}, \\
& \hat{t}_{\boldsymbol{y}}(\tilde{\boldsymbol{x}}, \boldsymbol{x})=-2(\tilde{\boldsymbol{y}}-y) \tilde{\boldsymbol{n}} \cdot(\tilde{\boldsymbol{x}}-\boldsymbol{x}) \hat{p}(\tilde{\boldsymbol{x}}, \boldsymbol{x}) / r^{2}
\end{aligned}
$$

where $\tilde{\boldsymbol{n}}=\left(\tilde{n}_{x}, \tilde{n}_{y}\right)$ is the outward normal at $\tilde{\boldsymbol{x}}$ on $\Gamma$.

Differentiation of (A.8) further gives

$$
\begin{aligned}
\hat{p}(\boldsymbol{x})= & -\hat{p}^{1}\left(\boldsymbol{x}_{s}, \boldsymbol{x}\right) f_{x}-\hat{p}^{2}\left(\boldsymbol{x}_{s}, \boldsymbol{x}\right) f_{y}-\int_{\Gamma}\left\{t_{x}(\tilde{\boldsymbol{x}}) \hat{p}^{1}(\tilde{\boldsymbol{x}}, \boldsymbol{x})+t_{y}(\tilde{\boldsymbol{x}}) \hat{p}^{2}(\tilde{\boldsymbol{x}}, \boldsymbol{x})\right\} \mathrm{d} \tilde{\Gamma} \\
& +2 \mu \int_{\Gamma}\left\{u(\tilde{\boldsymbol{x}}) \tilde{\boldsymbol{n}} \cdot \nabla \hat{p}^{1}(\tilde{\boldsymbol{x}}, \boldsymbol{x})+v(\tilde{\boldsymbol{x}}) \tilde{\boldsymbol{n}} \cdot \nabla \hat{p}^{2}(\tilde{\boldsymbol{x}}, \tilde{\boldsymbol{x}})\right\} \mathrm{d} \tilde{\Gamma}, \quad \boldsymbol{x} \in \Omega .
\end{aligned}
$$

For further details, see [12]. Notice that $\Gamma$ is composed of $\Gamma_{\mathrm{g}}^{ \pm}$and $\Gamma_{h}^{ \pm}$in our rectangle problem.

\section{Appendix B. A boundary element scheme in least-squares}

Since the fundamental solutions of (A.1) are divergent-free, we have

$$
0=\int_{\Omega}(\partial \hat{u} / \partial x+\partial \hat{v} / \partial y) \mathrm{d} \tilde{\Omega}
$$

which yields immediately

$$
0=\int_{\Gamma}\left(\tilde{n}_{x} \hat{u}+\tilde{n}_{y} \hat{v}\right) \mathrm{d} \tilde{\Gamma} .
$$

It is then obvious in (A.8) that the constant traction forces $t_{x}$ and $t_{y}$ do not influence upon the velocities. In the boundary element approximations for Stokes flow, therefore, we should extinguish these degrees of freedom on traction forces.

On the smooth part of $\Gamma$, let $u_{t}$ and $t_{n}$ denote the tangential velocity and normal traction force, respectively. At the smooth point $x$ on $\Gamma,(\mathrm{A} .8)$ can then be written for the tangential velocity as

$$
\frac{1}{2} u_{t}(x)=K_{t}\left[x, u_{t}(\tilde{x}), t_{n}(\tilde{x})\right],
$$


and for the normal velocity as

$$
0=K_{n}\left[\boldsymbol{x}, u_{t}(\tilde{\boldsymbol{x}}), t_{n}(\tilde{\boldsymbol{x}})\right]
$$

We then express the traction force balance conditions as

$$
\begin{aligned}
& C_{x}\left[\tilde{n}_{x} t_{n}(\tilde{\boldsymbol{x}})\right]=f_{x}+\int_{\Gamma} t_{x}(\tilde{\boldsymbol{x}}) \mathrm{d} \tilde{\Gamma}=0, \\
& C_{y}\left[\tilde{n}_{y} t_{n}(\tilde{\boldsymbol{x}})\right]=f_{y}+\int_{\Gamma} t_{y}(\tilde{\boldsymbol{x}}) \mathrm{d} \tilde{\Gamma}=0,
\end{aligned}
$$

Denoting the boundary element approximations by superscript $h$, we introduce a least-square functional of the form

$$
\begin{aligned}
\chi= & \frac{1}{2} \sum_{m}\left\{\frac{1}{2} u_{t}^{h}\left(\boldsymbol{x}_{m}\right)-K_{t}\left[\boldsymbol{x}_{m}, u_{t}^{h}(\tilde{\boldsymbol{x}}), t_{n}^{h}(\tilde{\boldsymbol{x}})\right]\right\}^{2}+\frac{1}{2} \sum_{l}\left\{K_{n}\left[\boldsymbol{x}_{l}, u_{t}^{h}(\tilde{\boldsymbol{x}}), t_{n}^{h}(\tilde{\boldsymbol{x}})\right]\right\}^{2} \\
& +\alpha_{x}\left\{C_{x}\left[\tilde{n}_{x} t_{n}^{h}(\tilde{\boldsymbol{x}})\right]\right\}^{2}+\alpha_{y}\left\{C_{y}\left[\tilde{n}_{y} t_{n}^{h}(\tilde{\boldsymbol{x}})\right]\right\}^{2}
\end{aligned}
$$

Here $\alpha_{x}$ and $\alpha_{y}$ are Lagrange multipliers, and the smooth points $x_{m}$ and $x_{l}$ are sampled on $\Gamma$ so that the sampled number is greater than or equal to that of the discretized variables to be determined plus two of Lagrange multipliers. Minimization of $\chi$ by (B.5) then determines $u_{t}^{h}$ and $t_{n}^{h}$.

It is emphasized that imposition of the constraints of (B.4) guarantees the uniqueness of our boundary element solutions.

\section{Acknowledgment}

The authors are grateful to Prof. Norio Takenaka of Nihon University, for his useful comments and suggestions.

\section{References}

[1] J.H. Argyris and G. Mareczek, Finite element analysis of slow incompressible viscous fluid motion, Ingr.-Arch. 43 (1974) 92-109.

[2] O.C. Zienkiewicz, The Finite Element Method (McGraw-Hill, London, 3rd ed., 1977).

[3] T.J. Chung, Finite Element Analysis in Fluid Dynamics (McGraw-Hill, New York, 1978).

[4] J.T. Oden, N. Kikuchi and Y.J. Song, A class of contact problems for linearly incompressible materials, TICOM Rept. 79, University of Texas at Austin, TX, 1979.

[5] N. Kikuchi, Convergence of a penalty-finite element approximations for an ohtotacle problem, TICOM Rept. 79, University of Texas at Austin, TX, 1979.

[6] J.N. Reddy, On the finite element method with penalty for incompressible fluid flow problem, in: J.R. Whiteman, ed., The Mathematics of Finite Elements and Applications 3 (Academic Press, London, 1979)
227-235. 
[7] N. Kikuchi and Y.J. Song, Contact problems involving forces and moments for incompressible linearly elastic materials, Internat. J. Engrg. Sci. 18 (1980) 357-377.

[8] J.T. Oden and O. Jacquotte, A stable second-order accurate finite element scheme for the analysis of two-dimensional incompressible viscous flows, TICOM Rept. 82, University of Texas at Austin, TX, 1982.

[9] N. Kikuchi, On a smoothing scheme of penalty/finite element methods for Stokes flow analysis, in: T. Kawai, ed., Finite Element Flow Analysis (University of Tokyo Press, Tokyo, 1982) 45-50.

[10] M. Okabe and N. Kikuchi, Some general Lagrange interpolations over simplex finite elements with reference to derivative singularities, Comput. Meths. Appl. Mech. Engrg. 28 (1981) 1-25.

[11] I. Babuška, B.A. Szabo and I.N. Katz, The p-version of the finite element method, SIAM J. Numer. Anal. 18 (1981) $515-545$.

[12] M. Okabe, A boundary element approach in the incompressible viscous flow, in: T. Kawai, ed., Finite Element Flow Analysis (University of Tokyo Press, Tukyo, 1982) 915-922.

[13] C.A. Brebbia, The Boundary Element Method for Engineer (Pentech, London, 1978).

[14] R.V. Churchill, Complex Variables and Applications (McGraw-Hill, New York, 1960).

[15] M. Okabe and N. Kikuchi, Analytical solutions of some steady-state electrical problems in the rectangular domain, Comput. Meths. Appl. Mech. Engrg. 36 (1983) 167-189.

[16] M. Okabe, Singularity constants and positioning constants in Poisson's equation with a point source of unit intensity, Comput. Meths. Appl. Mech. Engrg. 25 (1981) 287-297. 